\title{
Clinic experience in discoid lupus erythematosus: a retrospective study of 132 cases
}

\author{
Goknur Ozaydın Yavuz¹, Ibrahim Halil Yavuz¹, Irfan Bayram², Rojda Aktar¹, Serap Gunes Bilgili \\ 1Department of Dermatology, Faculty of Medicine, Van Yuzuncu Yil University, Van, Turkey \\ 2Department of Pathology, Faculty of Medicine, Van Yuzuncu Yil University, Van, Turkey
}

Adv Dermatol Allergol 2019; XXXVI (6): 739-743

DOI: https://doi.org/10.5114/ada.2018.77726

\begin{abstract}
Introduction: Lupus erythematosus (LE) is an autoimmune disease characterized by a broad range of cutaneous manifestations. Discoid LE (DLE) is the most common chronic manifestation of LE. Literature reviews show that there are a limited number of large-series studies investigating DLE. Additionally, there is still no consensus on the etiological factors of DLE such as sun exposure and smoking.

Aim: To evaluate the clinical and demographic characteristics of patients with DLE.

Material and methods: The study included patients who were hospitalized in the inpatient and outpatient clinics at the Dermatology Department. Age, gender, treatment method, history of smoking, antinuclear antibody positivity, progression to systemic lupus erythematosus (SLE), photosensitivity, and laboratory findings were recorded for each patient.

Results: The study included 132 patients comprising 67 (50.8\%) men and 65 (49.2\%) women. A family history was found in $3.8 \%$, SLE was detected in $5.3 \%$, and photosensitivity was revealed in $50.0 \%$ of the patients. ANA positivity was found in $23.7 \%$, a history of smoking was revealed in $61.4 \%$, and chronic sun exposure was detected in $42.4 \%$ of the patients.

Conclusions: Discoid LE, though identified long ago, remains unelucidated and there are very few studies in the literature reporting on DLE. The results indicated that smoking and chronic UV exposure are important risk factors for DLE. Moreover, although ANA positivity was high in our patients, the rate of progression to SLE was remarkably low. The results also showed that, contrary to common belief, there is no female preponderance in DLE.
\end{abstract}

Key words: discoid lupus erythematosus, smoking, antinuclear antibody.

\section{Introduction}

Lupus erythematosus (LE) is an autoimmune disease characterized by a broad range of cutaneous manifestations. After rheumatologic manifestation, cutaneous lupus erythematosus (CLE) is the second most common clinical manifestation of LE [1-4]. There are several distinct clinical presentations of CLE, mainly including acute CLE, subacute CLE (SCLE), and chronic CLE. Moreover, discoid LE (DLE) is another form which is the most common manifestation of CLE [2].

Discoid LE (DLE) is mostly seen in women in their $5^{\text {th }}$ and $6^{\text {th }}$ decades of life. DLE usually has a more benign course as compared to other CLE subtypes. Discoid LE often involves the head and neck, predominantly the hairy areas of the skin such as scalp. Involvement of hairy skin is seen in $60 \%$ of patients with DLE while isolated involvement of hairy skin is seen in only $10 \%$ of patients.
A previous study also reported that scarring alopecia was detected in $34 \%$ of patients with DLE $[1,3]$.

The etiopathogenesis of DLE is multifactorial. Of note, genetic and environmental causes and congenital and acquired immune response change have been reported as the leading causes of DLE. Moreover, ultraviolet (UV) exposure is regarded as the most common cause of DLE, followed by drugs, smoking, vitamin D deficiency, and HIV infection [1, 4-6]. On the other hand, 5-10\% of patients with DLE may progress to systemic lupus erythematosus (SLE), which shows that the localized form of DLE is not the only form [1].

The primary step in the treatment of DLE is prevention of the disease, which is dependent on a combination of patient education and avoidance of predisposing factors. Mainstay treatment includes topical steroids, topical calcineurin inhibitors, and antimalarial drugs [6, 7]. 
Although DLE was identified long ago, there are a limited number of large-series studies in the literature. Additionally, there is still no consensus on the etiological factors such as sun exposure and smoking.

\section{Aim}

In this study, we aimed to evaluate the clinical and demographic characteristics of patients with DLE.

\section{Material and methods}

The retrospective study included patients who were hospitalized in the inpatient and outpatient clinics at the

Table 1. Demographic characteristics of patients

\begin{tabular}{lcc}
\hline Parameter & Value & Percentage \\
\hline \begin{tabular}{l} 
Gender: \\
\cline { 2 - 2 } Male
\end{tabular} & 67 & 50.8 \\
\hline Female & 65 & 49.2 \\
\hline $\begin{array}{l}\text { Occupation: } \\
\text { Farmer }\end{array}$ & 20 & 15.2 \\
\hline $\begin{array}{l}\text { Housewife/unemployed } \\
\text { Civil servant or other }\end{array}$ & 40 & 54.5 \\
\hline Age [years] & $43.2 \pm 14.1$ & 30.3 \\
\hline Age at onset [years] & $39.3 \pm 14.1$ & 38.5 \\
\hline
\end{tabular}

${ }^{*}$ For quantitative values, mean $\pm S D$ was used instead of $n$ and median was used instead of $\%$.

Table 2. Clinical features of patients

\begin{tabular}{lccc}
\hline Parameter & & Number & Percentage \\
\hline Family history & No & 127 & 96.2 \\
\cline { 2 - 4 } & Yes & 5 & 3.8 \\
\cline { 2 - 4 } SLE & No & 125 & 94.7 \\
\hline Photosensitivity & Yes & 7 & 5.3 \\
\cline { 2 - 4 } & No & 66 & 50.0 \\
\hline ANA positivity & Yes & 66 & 50.0 \\
\cline { 2 - 4 } & No & 100 & 76.3 \\
\hline Smoking & Yes & 31 & 23.7 \\
\cline { 2 - 4 } & No & 51 & 38.6 \\
\hline Chronic & Yes & 81 & 61.4 \\
\hline sun exposure & No & 76 & 57.6 \\
\hline Comorbidity & Yes & 56 & 42.4 \\
\cline { 2 - 4 } & DM & 9 & 6.8 \\
\cline { 2 - 4 } & Hyperthyroidism & 3 & 2.3 \\
\cline { 2 - 4 } & HT & 24 & 18.2 \\
\hline SLE systemic & No & 96.7 \\
\hline
\end{tabular}

SLE - systemic lupus erythematosus, DM - diabetes mellitus, HT - hypertension, ANA - antinuclear antibody.
Medical School Dermatology Department due to DLE between January 2005 and December 2017. Medical records of the patients were obtained from hospital databases and then analyzed for age, gender, lesion site, treatment method, comorbidities, family history, history of smoking, antinuclear antibody (ANA) positivity, progression to SLE, photosensitivity, long-term UV exposure, and histopathological and laboratory findings. Discoid LE was diagnosed based on clinical, histopathological, and direct immunofluorescence (DIF) findings. Care was taken to choose clinically well-demarcated, coin-shaped, squamous plaques with adherent scales, follicular plugging, intermittent telangiectasia and peripheral hyperpigmentation. In older lesions, those with central atrophy and hypopigmentation were chosen. Typical histopathological features of DLE included orthokeratotic hyperkeratosis, follicular plugging, epidermal atrophy, dyskeratosis, basal cell degeneration, basement-membrane thickening, and perivascular and periadnexal mononuclear cell infiltrate. During the DIF examination, granular deposition of IgG and C3 at the dermoepidermal junction was also investigated. Exclusion criteria were age younger than 18 years, absence of discoid lupus diagnosis, and lack of medical records.

\section{Statistical analysis}

Data were analyzed using SPSS for Windows version 15.0 (SPSS Co., Chicago, IL, USA). Normal distribution of data was analyzed using histogram plots and the Kolmogorov-Smirnov test. Descriptive data were expressed as mean, standard deviation (SD), median, frequency, and percentage. Nonparametric groups were compared using Mann-Whitney $U$ and Kruskal Wallis tests. Categorical variables were compared using the $\chi^{2}$ test and Fisher's exact test. A $p$-value of $<0.05$ was considered significant.

\section{Results}

The study included 132 patients comprising 67 (50.8\%) men and 65 (49.2\%) women. Of the 132 patients, 72 (54.5\%) were either unemployed or housewives. Mean age was 43.2 \pm 14.1 years and mean age at onset was $39.3 \pm 14.1$ years (Table 1).

A family history was found in 3.8\%, SLE was detected in $5.3 \%$, and photosensitivity was revealed in $50.0 \%$ of the patients. ANA positivity was found in $23.7 \%$, a history of smoking was revealed in $61.4 \%$, and chronic sun exposure was detected in $42.4 \%$ of the patients. Hypertension ( $\mathrm{HT}$ ) was the most common comorbidity (18.2\%), followed by diabetes mellitus (DM) (6.8\%) and hyperthyroidism (2.3\%). No comorbidity was detected in $72.7 \%$ of the patients (Table 2).

An analysis on the treatment methods indicated that $99.2 \%$ of the patients underwent treatment with sunscreen sun protection factor (SPF), 90.9\% underwent 
topical corticosteroid therapy, 56.8\% underwent hydroxychloroquine therapy, $19.7 \%$ underwent topical calcineurin therapy, and the remaining $2.3 \%$ received other therapies. On the other hand, hairy skin involvement was detected in $22.7 \%$, facial involvement was detected in $78.8 \%$, and whole body involvement was detected in $15.2 \%$ of the patients. A positive DIF finding was detected in $86.4 \%$ of the patients (Table 3 ).

A comparison between gender and family history, site of involvement, and treatment methods revealed that ANA positivity was higher in women than in men (32.3\% vs. 15.2\%) ( $p=0.021)$. However, no significant difference was found between gender and other variables $(p>0.05)$ (Table 4).

The 12-year incidence rate over the period 2005-2017 was 12.62 cases per 100,000 population and the estimated annual incidence rate was 1.05 cases per 100,000 population.

\section{Discussion}

The results indicated that DLE has a low incidence in the eastern part of Turkey and, contrary to common belief, there is no female preponderance in DLE. To our knowledge, there are very few studies in the literature reporting on the incidence rate of DLE. Durosaro et al. reported the annual incidence rate of DLE as 4.3/100,000 population while Gronhagen et al. found a rate of $1.05 / 100,000$ population. In our study, we found a rate of $1.05 / 100,000$ population, which was lower than that of other studies $[8,9]$.
Table 3. Treatment methods and sites of involvement

\begin{tabular}{|c|c|c|c|}
\hline Parameter & & Number & Percentage \\
\hline \multirow[t]{2}{*}{ SPF } & No & 1 & 0.8 \\
\hline & Yes & 131 & 99.2 \\
\hline \multirow[t]{2}{*}{ Topical corticosteroid } & No & 12 & 9.1 \\
\hline & Yes & 120 & 90.9 \\
\hline \multirow[t]{2}{*}{ Hydroxychloroquine } & No & 57 & 43.2 \\
\hline & Yes & 75 & 56.8 \\
\hline \multirow{2}{*}{$\begin{array}{l}\text { Topical calcineurin } \\
\text { inhibitor }\end{array}$} & No & 106 & 80.3 \\
\hline & Yes & 26 & 19.7 \\
\hline \multirow[t]{2}{*}{ Others } & No & 129 & 97.7 \\
\hline & Yes & 3 & 2.3 \\
\hline \multirow[t]{2}{*}{ Hairy skin* } & No & 102 & 77.3 \\
\hline & Yes & 30 & 22.7 \\
\hline \multirow[t]{2}{*}{ Face $^{*}$} & No & 28 & 21.2 \\
\hline & Yes & 104 & 78.8 \\
\hline \multirow[t]{2}{*}{ Whole body* } & No & 112 & 84.8 \\
\hline & Yes & 20 & 15.2 \\
\hline \multirow[t]{2}{*}{ DIF test } & Negative & 18 & 13.6 \\
\hline & Positive & 114 & 86.4 \\
\hline
\end{tabular}

SPF - sun protection factor, DIF-direct immunofluorescence, *involvement.

Studies have indicated that DLE is more common in women than in men. Of note, the female-to-male ratio was reported as 3.2/1 by Koskenmies et al. and as 5/1 by both Jacyk and Damisah, and Insawang et al. In our

Table 4. Gender-based comparison of family history, site of involvement, and treatment methods

\begin{tabular}{|c|c|c|c|c|c|c|}
\hline \multirow[t]{3}{*}{ Parameter } & & \multicolumn{4}{|c|}{ Gender } & \multirow{3}{*}{$P$-value } \\
\hline & & \multicolumn{2}{|c|}{ Male } & \multicolumn{2}{|c|}{ Female } & \\
\hline & & $n$ & $\%$ & $n$ & $\%$ & \\
\hline \multirow[t]{2}{*}{ Family history } & No & 65 & 97.0 & 62 & 95.4 & $0.678^{b}$ \\
\hline & Yes & 2 & 3.0 & 3 & 4.6 & \\
\hline \multirow[t]{2}{*}{ Systemic lupus erythematosus } & No & 65 & 97.0 & 60 & 92.3 & $0.270^{b}$ \\
\hline & Yes & 2 & 3.0 & 5 & 7.7 & \\
\hline \multirow[t]{2}{*}{ Photosensitivity } & No & 38 & 56.7 & 28 & 43.1 & $0.117^{\mathrm{a}}$ \\
\hline & Yes & 29 & 43.3 & 37 & 56.9 & \\
\hline \multirow[t]{2}{*}{ ANA positivity } & No & 56 & 84.8 & 44 & 67.7 & $0.021^{\mathrm{a}}$ \\
\hline & Yes & 10 & 15.2 & 21 & 32.3 & \\
\hline \multirow[t]{2}{*}{ Hairy skin* } & No & 50 & 74.6 & 52 & 80.0 & $0.461^{a}$ \\
\hline & Yes & 17 & 25.4 & 13 & 20.0 & \\
\hline \multirow[t]{2}{*}{ Face* $^{*}$} & No & 17 & 25.4 & 11 & 16.9 & $0.235^{a}$ \\
\hline & Yes & 50 & 74.6 & 54 & 83.1 & \\
\hline \multirow[t]{2}{*}{ Whole body* } & No & 55 & 82.1 & 57 & 87.7 & $0.369^{a}$ \\
\hline & Yes & 12 & 17.9 & 8 & 12.3 & \\
\hline Duration of disease & & 2.0 & $0.1-11.0$ & 2.0 & $0.1-12.0$ & $0.080^{c}$ \\
\hline
\end{tabular}

${ }^{a} \chi^{2}$ test; ${ }^{b}$ Fisher's exact test; *involvement; for quantitative values, median was used instead of $n$ and minimum-maximum values were used instead of \%; 'Mann-Whitney U test. 
study, the incidence of DLE in men and women was almost equal, which, contrary to common belief, suggests that there is no female preponderance in $\operatorname{DLE}[6,10,11]$.

Discoid LE is mostly seen between the ages of 40 and 60 years and the mean age at onset was reported to be 42 years by Koskenmies et al. and 38 years by $\mathrm{Ng}$ et al. $[10,12]$. Similarly, we also found that the mean age at onset was 39.3 years, which was consistent with the literature. Taken together, these findings indicate that DLE has a peak incidence in the third to fourth decades of life. Nevertheless, apart from these studies, there are also several pediatric studies with large patient series [13].

Discoid LE mostly involves the face and hairy areas of the skin. Tang et al. reported that the face was the most common site of involvement as opposed to hairy areas of the skin in the study by Fahad et al. [7, 14-16]. In our study, the rate of facial involvement was higher than that of other sites, which implies the presence of photodermatosis.

Literature reviews indicate that there are contradictory rates of photosensitivity in patients with DLE. Insawang et al. reported a rate of $14.6 \%$ and $71.3 \%$ in two different studies while Callen reported a rate of $87 \%$. In contrast, we found a rate of $50 \%$, which was an average rate when compared to the rates reported in the literature. All these rates imply that the incidence of photosensitivity is seriously high in DLE and thus protection from sunlight is highly important in patients with $\operatorname{DLE}[6,10,17]$.

Although DLE is known to be aggravated by sun exposure, chronic sun exposure is not mentioned in most studies. In our study, chronic sun exposure was detected in $42.4 \%$ of the patients, which suggests the importance of the cumulative effect of sunlight exposure in DLE. Moreover, studies have also shown that UVB could be a more important factor than UVA in DLE [7].

Some cases of DLE may progress to SLE or may coexist with both DLE and SLE. The rate of progression to SLE was reported as $20.7 \%$ by Insawang et al. and as $17 \%$ by Tang et al. On the other hand, the rate of coexistence of DLE and SLE was reported as $9.8 \%$ by Grönhagen et al. and $6.5 \%$ by Callen $[6,7,14,17]$. In our study, we found that the rate of coexistence was $5.3 \%$, which was lower than the rates reported in the literature. However, taken together, all these rates imply that only a low rate of patients with DLE progress to SLE. Moreover, compared to acute and subacute LE, DLE appears to provide protection against progression to SLE since the acute and subacute forms of DLE lead to high rates of progression $[1,6,7]$.

Antinuclear antibodies have been shown to be highly important in patients with DLE and to be a potential indicator of progression to SLE. ANA positivity was detected in $16.1 \%$ of the patients evaluated by Fahad et al. and in $67 \%$ of the patients evaluated by Tang et al. [14, 15]. In our study, ANA positivity was detected in $23.7 \%$ of the patients. Although this rate was similar to the rate reported by Fahad et al., it was lower than the rate reported by Tang et al., which probably resulted from the relatively smaller patient series evaluated by Tang et al. Moreover, the rate of progression to SLE (5.3\%) and ANA positivity $(23.7 \%)$ in our study suggests that the ANA positivity in DLE is not associated with the presence of SLE. However, the significantly high rate of ANA positivity in our female patients suggests that this parameter is likely to differ between genders.

There are contradictory rates of family history in patients with DLE in the literature. While Sampaio et al. found a rate of $11.8 \%$, Insawang et al. reported that no family history was detected in any patient $[6,13]$. In our study, however, a family history was detected in 5 (3.8\%) patients, which suggests the presence of a genetic tendency.

Smoking has been implicated in the etiopathology of DLE in some studies. Miot et al., for instance, reported that $84.2 \%$ of patients and $33.5 \%$ of control subjects had a history of smoking, also noting that the rate of smoking was higher in the patients with DLE and smoking is a leading factor aggravating DLE [18]. Similarly, Böckle et al. found that there was a high rate of smoking among patients with DLE and lupus erythematosus tumidus (LET) [19]. In line with the literature, the smoking rate in our patients was $61.4 \%$, which suggests that complete cessation of smoking is imperative in patients with DLE.

The first step in the treatment of discoid lupus erythematosus is high potency topical corticosteroids. In a previous study, topical steroid treatment was given to all patients with discoid lupus. However, some of the patients did not respond to treatment [6]. In another study, $60 \%$ of patients received topical corticosteroid treatment [20]. In our study, $90.1 \%$ of patients received topical corticosteroid therapy. This was consistent with the literature.

Our study was limited since it was a single-center retrospective study. Additionally, no anti-double stranded DNA (anti-dsDNA) test was performed in some patients due to technical difficulties, and the vitamin D level was not measured in any patient.

\section{Conclusions}

Discoid LE, though identified many years ago, remains unelucidated. Moreover, there are very few studies in the literature reporting on DLE. The results of this study indicated that the estimated annual incidence rate of DLE in the eastern part of Turkey is 1.05 cases per 100,000 population and smoking and chronic UV exposure are important risk factors for DLE. Moreover, although ANA positivity was high in our patients, the rate of progression to SLE was remarkably low. The results also showed that, contrary to common belief, there is no female preponderance in DLE. Further prospective multicenter studies with large patient series are needed to better understand the clinical features of DLE. 


\section{Conflict of interest}

The authors declare no conflict of interest.

\section{References}

1. Okon LG, Werth VP. Cutaneous lupus erythematosus: diagnosis and treatment. Best Pract Res Clin Rheumatol 2013; 27: 391-404.

2. Walling HW, Sontheimer RD. Cutaneous lupus erythematosus: issues in diagnosis and treatment. Am J Clin Dermatol 2009; 10: 365-81.

3. Rothfield N, Sontheimer RD, Bernstein M. Lupus erythematosus: systemic and cutaneous manifestations. Clin Dermatol 2006; 24: 348-62.

4. Two A, So JK, Paravar T. Discoid lupus and human immunodeficiency virus: a retrospective chart review to determine the prevalence and progression of co-occurrence of these conditions at a single academic center. Indian J Dermatol 2017; 62: 226

5. Kirchhof MG, Dutz JP. The immunopathology of cutaneous lupus erythematosus. Rheum Dis Clin North Am 2014; 40: 455-74.

6. Insawang M, Kulthanan K, Chularojanamontri L, et al. Discoid lupus erythematosus: description of 130 cases and review of their natural history and clinical course. J Clin Immunol Immunopathol Res 2010; 2: 1-8.

7. Grönhagen CM, Nyberg F. Cutaneous lupus erythematosus: an update. Indian Dermatol Online J 2014; 5: 7-13.

8. Durosaro O, Davis MD, Reed KB, et al. Incidence of cutaneous lupus erythematosus, 1965-2005: a population-based study. Arch Dermatol 2009; 145: 249-53.

9. Grönhagen CM, Fored CM, Granath F, et al. Cutaneous lupus erythematosus and the association with systemic lupus erythematosus: a population-based cohort of 1088 patients in Sweden. Br J Dermatol 2011; 164: 1335-41.

10. Koskenmies S, Jarvinen TM, Onkamo P, et al. Clinical and laboratory characteristics of Finnish lupus erythematosus patients with cutaneous manifestations. Lupus 2008; 17: 337-47.

11. Jacyk WK, Damisah M. Discoid lupus erythematosus in the Nigerians. Br J Dermatol 1979; 100: 131-5.

12. Ng SK, Ratnam KV, Tan T. Discoid lupus erythematosus in Singapore. Singapore Med J 1985; 26: 365-8.

13. Sampaio MC, de Oliveira ZN, Machado MC, et al. Discoid lupus erythematosus in children: a retrospective study of 34 patients. Pediatr Dermatol 2008; 25: 163-7.

14. Tang WYM, Chan LY, Lo KK. Discoid lupus erythematosus in Hongkong Chinese: a review of 12 cases. HKMJ 1996; 2: 239-45.

15. Al-Saif FM, Al-Balbeesi AO, Al-Samary Al, et al. Discoid lupus erythematosus in a Saudi population: clinical and histopathological study. I Saudi Soc Dermatol Dermatol Surg 2012; 16: 9-12.

16. Bajaj DR, Devrajani BR, Matlani BL. Discoid lupus erythematosus: a profile. J Coll Physicians Pak 2010; 20: 361-4.

17. Callen JP. Cutaneous lupus erythematosus: a personal approach to management. Australas I Dermatol 2006; 47: 13-27.

18. Miot HA, Bartoli Miot LD, Haddad GR. Association between discoid lupus erythematosus and cigarette smoking. Dermatology 2005; 211: 118-22.

19. Böckle BC, Sepp NT. Smoking is highly associated with discoid lupus erythematosus and lupus erythematosus tumidus: analysis of 405 patients. Lupus 2015; 24: 669-74.
20. Moises-Alfaro C, Berrón-Pérez R, Carrasco-Daza D, et al. Discoid lupus erythematosus in children: clinical, histopathologic, and follow-up features in 27 cases. Pediatr Dermatol 2003; 20: 103-7. 